\title{
Tableros de partículas de bambú (Bambusa vulgaris Schrad) y resina poliuretana a base de aceite de rícino (Ricinus communis L.)
}

\author{
Boards made of bamboo (Bambusa vulgaris \\ Schrad) particles and castor oil-based \\ polyurethane resin
}

\section{Flávio Januário José Antonio Ludovico Beraldo}

\section{Resumen

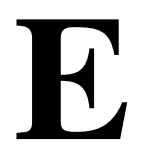

sta investigación tiene como objetivo la elaboración y la evaluación de tableros de partículas homogéneas aglomeradas, utilizándose dos materiales alternativos en la búsqueda de la sustentabilidad del proceso productivo. Fueron utilizadas partículas de Bambusa vulgaris Schrad por la rapidez del ciclo de producción de esta especie de bambú. Como pegante fue utilizada la resina poliuretana a base de aceite de ricino (Ricinus communis L.), por su origen parcialmente renovable, y por ser considerada no tóxica. Para la caracterización del material, fueron fabricadas, en escala de laboratorio, tableros de partículas de bambú con dimensiones inferiores al $2.4 \mathrm{~mm}$, combinadas con 5\%, $10 \%$ y $15 \%$ de resina en relación a la masa de partículas de bambú. Probetas fueron evaluadas de acuerdo con las especificaciones de la norma NBR 14810 Tableros de madera aglomerada. Antes de los ensayos de compresión las probetas fueron evaluadas por medio del ensayo no destructivo por ultra-sonido. Los resultados obtenidos indicaron que los tableros con el contenido de $10 \%$ de resina no fue estadísticamente diferente al contenido de $15 \%$, siendo ambos superiores al contenido de $5 \%$. La mayoría de las propiedades de los tableros fueron inferiores a aquellas de los tableros comerciales. No fue posible correlacionar la velocidad del pulso de ultra-sonido con la resistencia a la compresión longitudinal.

Flávio Januário José Faculdade de Engenharia Agrícola Universidade Estadual de Campinas Cidade Universitária Zeferino Vaz, Av. Candido Rondon, 501, Barão Geraldo

Campinas - SP - Brasil CEP 13083-875

Tel.: (55) 19-35211030 E-mail:

casanarvore@hotmail.com.br

Antonio Ludovico Beraldo Faculdade de Engenharia Agrícola Universidade Estadual de Tel. (55) 19-35211085 E-mail: beraldo@agr.unicamp.br

Recebido em 19/11/09 Aceito em 01/08/10
Palavras-chave: Normas. Aceite de rícino. Bambusa vulgaris. Ensayos no destructivos. Poliuretano. Tableros de partículas.

\begin{abstract}
The aim of this study was to manufacture and evaluate particleboards made with two alternative materials, seeking to develop a sustainable production process. Particles from Bambusa vulgaris Schrad were used because of the fast production cycle of this species. The binder used was castor oil (Ricinus communis L.) based polyurethane resin, since it is partially produced by a renewable source and it is considered to be non-toxic. For the characterization of the material, boards made of bamboo particles smaller than $2.4 \mathrm{~mm}$, combined with $5 \%, 10 \%$ and $15 \%$ resin content in relation to the bamboo-particle mass, were manufactured at laboratory scale. Specimens were evaluated according to the Brazilian standard NBR 14810 - agglomerate wood boards. Before the longitudinal compression tests, the specimens were tested by means of a non-destructive ultrasound evaluation. The results showed that the boards with $10 \%$ resin content were not statistically different from those with $15 \%$ resin content, and both were superior to boards with $5 \%$ resin content. Most of the board properties were inferior to those of commercial boards. It was not possible to correlate ultrasonic pulse velocity across the specimens with longitudinal compression strength.
\end{abstract}

Keywords: Castor oil. Bambusa vulgaris. Non-destructive tests. Polyurethane. Particleboards. 


\section{Introducción}

La producción de los tableros de partículas aglomeradas, de acuerdo a (KOLLMANN; KUENZI; STAMM, 1975), empezó durante el periodo de la segunda guerra mundial, debido a la escasez de madera natural en la Alemania.

Con una capacidad de producción de aglomerados, MDF y tableros duros de 8.5 millones de $\mathrm{m}^{3}$ al año, la exportación del sector brasileño en 2003 fue de $32838 \mathrm{~m}^{3}$, generando 30 mil puestos de trabajo (ASSOCIAÇÃO..., 2010).

Actualmente las empresas brasileñas con mayor capacidad productiva de tableros emplean como materia prima, partículas originarias de los géneros Pinus y Eucalyptus. El aglomerante más utilizado en la fabricación de los tableros es la ureaformaldehido (ELEOTÉRIO, 2000). Esta resina presenta dos inconvenientes: una limitada resistencia a la humedad y de acuerdo con (MALONEY, 1996; PIZZI, 1994), debido a la emisión de formaldehido, se trata de un producto potencialmente cancerígeno.

Como alternativa para la producción de las partículas, el bambú muestra algunos aspectos en que supera al pino y el eucalipto. Una de las ventajas se debe a que el bambú puede ser cosechado en prácticamente todas las regiones brasileñas. Esta planta presenta ciclos de cosecha muy cortos, entre dos a tres años para la especie Bambusa vulgaris (PEREIRA; BERALDO, 2007). Esta especie es cultivada en grandes áreas del nordeste del país, suministrando materia-prima para la fabricación de papel y celulosa (ITAPAGÉ, 2004). En caso del pino y del eucalipto, para la producción de las partículas para tableros los ciclos son de 10 y 7 años, respectivamente (SBS, 2004).

La crisis del petróleo ha impulsado la búsqueda de soluciones alternativas en los diferentes campos de aplicación de esa materia prima. En el caso específico de la industria maderera los adhesivos a base de derivados de petróleo son responsables por cerca de $60 \%$ del costo de fabricación de los tableros.

La planta denominada Mamona o Ricino (Ricinus communis L.) es una planta originaria de la India e pertenece a la familia Euphorbiaceae. En Brasil fue introducida en el siglo XVI, durante el periodo colonial, para producir aceite para iluminación en las viviendas de los esclavos. Los cultivares de Mamona existentes en Brasil presentan ciclos de producción entre los 180 al 240 días; la productividad promedia es evaluada en $1500 \mathrm{~kg} / \mathrm{ha}$ con posibilidad de alcanzar los $4000 \mathrm{~kg} / \mathrm{ha}$. De las semillas se extrae cerca de $47 \%$ del aceite (www.iac.sp.gov.br). El aceite aún tenga acción fungicida $\mathrm{y}$ bactericida también puede ser empleado en varias aplicaciones: medicamentos, tintas, barnices, cosméticos, lubrificantes especiales y en la producción de combustible alternativo (reciente programa energético brasileño propuso la sustitución del $2 \%$ al $5 \%$ del diesel por biodiesel de aceites vegetales). Investigaciones desarrolladas por el Prof. Gilberto Chierice en el Instituto de Química de la Universidad de San Pablo (USP - San Carlos), fueran exitosas y se obtuvo un adhesivo poliuretano a base de aceite de ricino (APAR). Ese polímero fue testado en implantes quirúrgicos y odontológicos, presentando varias ventajas en comparación con los materiales convencionales (resistencia, maleabilidad, ligereza, estabilidad y ausencia de las toxinas).

En el Departamento de Maderas de la USP - San Carlos varias investigaciones analizaron la aplicación del APAR, como una alternativa a los adhesivos convencionales derivados del petróleo, en la fabricación de tableros de partículas (DIAS et al., 2004; CAMPOS; LAHR, 2004), contrachapados (DIAS; LAHR, 2004) y madera laminada colada (JESUS, 2000; AZAMBUJA, 2002). Otros investigadores analizaron el comportamiento del APAR con fibras vegetales (MOTHÉ; ARAÚJO, 2004) o artificiales (FIORELLI, 2002).

Los tableros de partículas posibilitan la manufactura de muebles, pisos y revestimientos, sin necesitar la tala de grandes árboles, los cuales requieren un largo tiempo para alcanzar las dimensiones necesarias para la obtención de tableros.

Este trabajo tiene como objetivo la manufactura y la evaluación de tableros de partículas de bambú aglomeradas con resina poliuretana bi-componente a base de aceite de ricino. Las siguientes etapas fueron desarrolladas:

(a) evaluación de la influencia de tres contenidos de resina poliuretana bi-componente a base de aceite de ricino $(5 \%, 10 \%$ y $15 \%$, con relación a la masa de partículas de bambú);

(b) comparación de los valores obtenidos en los ensayos de los tableros de partículas de bambú, con los estándares de la NBR 14810 (ABNT, 2002b);

(c) comparación estadística de los tableros que presentaron los resultados más adecuados, con tableros de partículas aglomeradas adquiridos comercialmente; y 
(d) evaluación, por medio del ensayo no destructivo (END) por ultra-sonido, del efecto de los contenidos de resina en la velocidad del pulso del ultrasonido (VPU) para verificar su posible correlación con la resistencia a la compresión longitudinal.

\section{Materiales y métodos}

\section{Partículas de bambú}

Para el desarrollo de la parte experimental del trabajo fueron utilizadas partículas provenientes de tallos de bambú de la especie Bambusa vulgaris, extraídos del bosque ubicado en el área experimental de la Facultad de Ingeniería Agrícola de la Universidad Estatal de Campinas - Unicamp. Los tallos fueron desintegrados en un equipo agrícola acoplado a un tractor. Luego, las partículas fueron secadas al aire durante una semana.

\section{Resina poliuretana bi-componente a base del aceite de ricino}

La resina poliuretana bi-componente a base del aceite de ricino fue proveída por una empresa de São Carlos - SP.

\section{Obtención de las partículas de bambú}

Las partículas fueron obtenidas de tallos maduros de bambú (más de 3 años de edad), siendo eliminadas preliminarmente las ramas y las hojas. Después del secado al aire (contenido de humedad alrededor de los 8\%), las astillas de bambú fueron trituradas en un equipo agrícola, seleccionándose las partículas con espesor máximo de $3 \mathrm{~mm}$ (abertura del tamiz).

Las partículas obtenidas fueron tamizadas y separadas en las aberturas de $2.40 \mathrm{~mm}, 0.42 \mathrm{~mm}$ y $0.149 \mathrm{~mm}$. Las partículas entre $2.40 \mathrm{~mm}$ y 0.42 $\mathrm{mm}$ fueron seleccionadas para la manufactura de los tableros.

\section{Manufactura de los tableros}

Fueron manufacturados diez tableros de dimensiones $40 \mathrm{~cm}$ x $40 \mathrm{~cm} \mathrm{x} 1.5 \mathrm{~cm}$ para cada contenido de resina $(5 \%, 10 \%$ y $15 \%)$. El procedimiento adoptado fue descrito anteriormente por Nascimento (2003).

\section{Aplicación de la resina a las partículas de bambú}

La resina fue sintetizada de acuerdo con las recomendaciones de la empresa KEHL (KEHL,
2004), mezclándose el pre-polímero (una parte en masa) con el poliól (dos partes en masa). A continuación, se adicionó la resina a las partículas de bambú, y se mezcló manualmente, utilizando de un guante de látex, como protección, por $5 \mathrm{~min}$.

Después de esta pre-homogeneización, la mezcla de resina con las partículas de bambú fue colocada en una batidora y mezclada por más 5 minutos.

\section{Formación del colchón de partículas de bambú y resina}

El colchón de la mezcla fue sometido a un preprensado en frío. El proceso fue realizado con el auxilio de una formadora de colchón, constituida de una caja de madera, sin tapa y sin fondo, cuya base presentaba dimensiones iguales a aquellas de los tableros $(40 \mathrm{~cm} \mathrm{x} 40 \mathrm{~cm})$.

\section{Prensado}

El colchón de partículas fue prensado con una temperatura constante de $60{ }^{\circ} \mathrm{C}$. Los tableros fueron protegidos con una película de papel aluminio para evitar su adherencia a los platos de la prensa.

En la primera etapa del prensado, se aplicó una presión de $4 \mathrm{MPa}$, por 10 minutos.

La segunda etapa fue iniciada aliviándose la presión hasta obtener un valor nulo. Este alivio se realizó en 5 minutos, y su objetivo fue provocar la liberación de los gases.

\section{Regularización}

Después del prensado, los tableros fueron aserrados para eliminar $5 \mathrm{~cm}$ de los bordes, como exigencia de la NBR 14810 (ABNT, 2002a, 2002b, 2002c) para la preparación de los ensayos, y para obtener una mejor calidad del producto finalizado.

\section{Tableros comerciales}

Para efecto de comparación, en una tienda comercial, se adquirieron tableros de $1.5 \mathrm{~cm}$ de espesor.

\section{Métodos de ensayos}

Los ensayos de caracterización de los tableros secados al aire (contenido de humedad alrededor de los 8\%) y la cantidad de probetas extraídas obedecieron a los estándares de la NBR 14810Tableros de madera aglomerada - Parte 3 métodos de ensayo.

Se efectuaron los siguientes ensayos: control de espesor; densidad; absorción de agua ( 2 h y 24 h); 
hinchamiento ( 2 h y 24 h); extracción de tornillo (en la superficie y en el canto); dureza Janka; (Figura 1a); compresión longitudinal (Figura 1b); flexión estática (Figura 1c) y tracción perpendicular.

Las probetas destinadas al ensayo de flexión estática fueron anteriormente evaluadas por medio de ensayo no destructivo por ultrasonido. En el aparato Ultrasonic Tester BP-7, disponiendo de sensores electro-acústicos de sección exponencial de $45 \mathrm{kHz}$ de frecuencia de resonancia, se determinaba el tiempo para la propagación de la onda y se obtenía la velocidad del pulso de ultrasonido (VPU). Los sensores fueron posicionados en el punto medio del espesor del tablero. Fue evaluada la constante elástica en la dirección longitudinal $\left(\mathrm{C}_{\mathrm{LL}}\right)$ por medio de la Ecuación 1:

$C_{L L}=\rho . V P U^{2} \cdot 10^{-9}$

Ec. 1

Siendo:

$\mathrm{C}_{\mathrm{LL}}=$ constante elástica dinámica $(\mathrm{GPa})$;

$\rho=$ masa específica aparente $\left(\mathrm{kg} / \mathrm{m}^{3}\right)$;

VPU $=$ velocidad del pulso de ultrasonido $(\mathrm{m} / \mathrm{s})$.

Además, se evaluó la posible dependencia entre la resistencia a la compresión y la VPU.

\section{Resultados y discusión}

El procedimiento experimental seguido durante la operación de prensado fue exactamente el mismo para todos los ensayos realizados, incluyendo la misma masa de material a prensar y un mismo operador para la preparación y ejecución de cada ensayo.

El único factor que fue variado fue el porcentaje en masa del pegamento añadido.

Así pues, las variaciones observadas, en una misma propiedad medida en los tableros resultantes del prensado en las condiciones antes descritas son atribuidas exclusivamente a un solo factor: la diferencia en dosificación del poliuretano.

La dosis de pegamento añadido provoca efectos de diferentes magnitudes en las propiedades bajo estudio que fueron evaluadas a través de un análisis de varianza donde se determinó, con una superioridad superior al $95 \%$ en la casi totalidad de los casos, en que medida la dosificación utilizada influye sobre la propiedad analizada y cual es el orden de efectividad de las diferentes dosis.

El indicador del grado de influencia del factor analizado (dosificación del poliuretano) sobre las propiedades medidas en los tableros prensados (módulo de rotura - MOR, densidad, absorción etc.) es el $\mathrm{p}$-valor. Cuando $\mathrm{p} \leq 0.05$ se puede concluir que el factor tiene una influencia significativa sobre la propiedad correspondiente. Por otro lado, cuando el p-valor es mucho menor que $0.05 \quad(p<0.05)$ se dice que el factor es altamente significativo, lo que equivale a certeza total de que el factor prácticamente determina el valor de la propiedad habido cuenta de que el resto de los factores permanezca constante (temperatura, tiempo y presión de prensado, masa de material a prensar etc.).

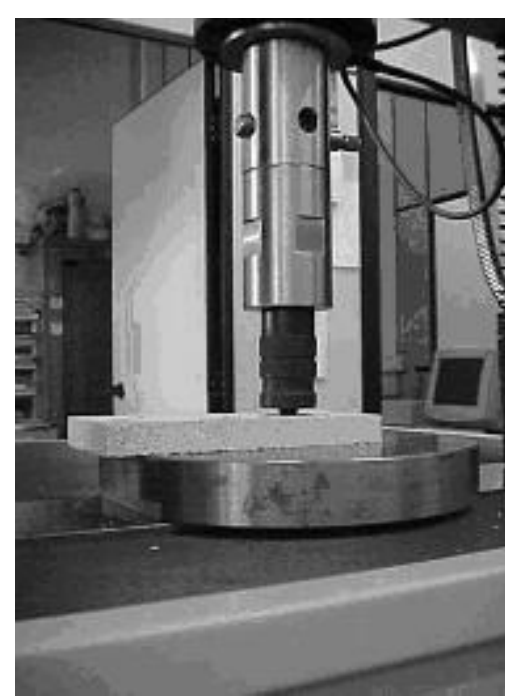

(a)

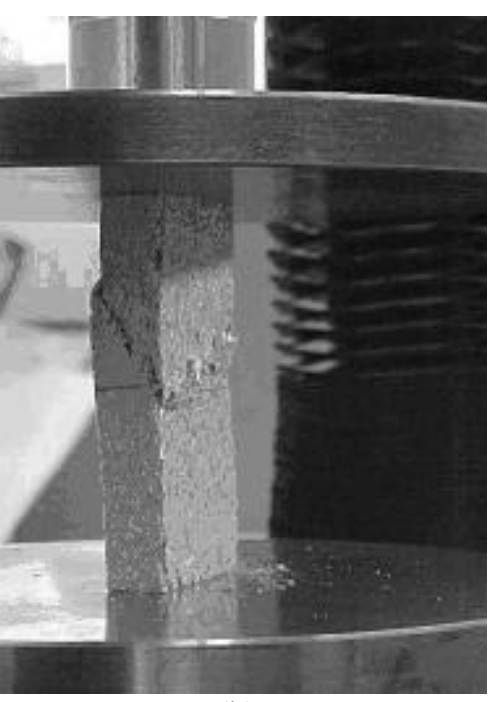

(b)

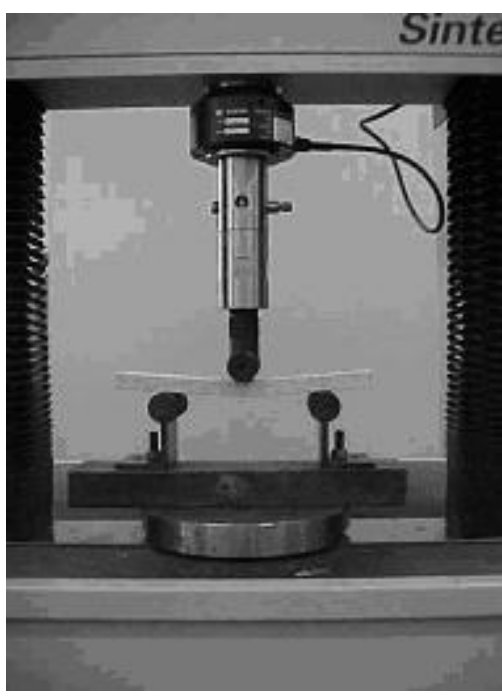

(c)

Figura 1 - (a) Dureza Janka, (b) compresión longitudinal y (c) flexión estática 
En la Tabla 1, la tracción perpendicular es la única propiedad que no presenta una altísima dependencia de la dosis de pegamento utilizado. Aún así, en ese caso el p-valor es solo ligeramente superior a $0.05(\mathrm{p}=0.0561)$ y por esa causa debe ser considerado como significativo. Ese resultado probablemente es debido a deficiencias experimentales observadas durante los ensayos correspondientes.

El resto de las propiedades consideradas evidencia una dependencia entre alta y altísima con respecto a la dosis de pegamento.

La Tabla 1 muestra el orden de efectividad de las diferentes dosis de pegamento, así como los valores medios de la propiedad bajo análisis debido a las diferentes dosificaciones.

Cuando esos valores promedios son estadísticamente indistinguibles aparece un signo de igual a (=) entre los promedios correspondientes.

Otra conclusión extraída de la Tabla 1 es que existe una diferencia significativa entre la dosis de $5 \%$ y las dosis de 10 y $15 \%$, siendo estas últimas de efectividades estadísticamente iguales sobre la mayoría de las propiedades analizadas.
El análisis estadístico de los resultados presentados en la Tabla 1 indicó no existir diferencia significativa (test de Tukey al 5\% de probabilidad) entre los contenidos de resina de $10 \%$ y de $15 \%$, ambos superiores al contenido de $5 \%$ en la mayoría de las propiedades evaluadas (densidad, absorción después de 24 h de inmersión en agua, hinchamiento después de $24 \mathrm{~h}$, extracción de tornillo en el canto y en la superficie, dureza Janka, compresión longitudinal y flexión estática). No obstante, hubo diferencia significativa (test de Tukey al 5\% de probabilidad) de los contenidos de resina en los resultados de espesor, absorción después de 2 h e hinchamiento después de $2 \mathrm{~h}$. Los datos obtenidos en el ensayo de tracción perpendicular de los tableros no pudieron ser adecuadamente considerados en el análisis. Aparentemente el exceso de resina en la superficie de los tableros impidió su adecuada adherencia con la placa metálica del aparato, observándose en varios ensayos su separación. Una alternativa posible es el empleo de garras específicas utilizadas en ensayos aplicados a los polímeros. Mientras tanto, algunas probetas lograron alcanzar valores superiores al de la norma (0.47 MPa), antes de ocurrir la pérdida de adherencia con la placa metálica.

\begin{tabular}{|c|c|c|c|}
\hline $\begin{array}{l}\text { Propiedad } \\
\text { Evaluada } \\
\end{array}$ & p-valor & $\begin{array}{l}\text { Evaluación } \\
\text { estadística }\end{array}$ & $\begin{array}{c}\text { Valores medios de las propiedades } \\
\text { y orden de efectividad de la } \\
\text { dosificación de pegamento }\end{array}$ \\
\hline Absorción (\%) - 2 h & 0.0000 & as & $15 *(27.69)<10(39.33)<5(78.98)$ \\
\hline Absorción (\%) - 24 h & $0.0600 * *$ & $\mathrm{~s}$ & $15(57.58)<10(60.46)<5(79.06)$ \\
\hline $\begin{array}{c}\text { Hinchamiento (\%) en espesor - } \\
2 \mathrm{~h}\end{array}$ & 0.0000 & as & $15(5.03)<10(7.76)<5(13.87)$ \\
\hline $\begin{array}{c}\text { Hinchamiento (\%) en espesor - } \\
24 \mathrm{~h}\end{array}$ & 0.0060 & as & $15(9.04)<10(11.48)<5(18.41)$ \\
\hline Espesor $(\mathbf{m m})$ & 0.0000 & as & $10(15.34)<15(15.95)<5(16.78)$ \\
\hline Densidad $\left(\mathrm{kg} / \mathrm{m}^{3}\right)$ & 0.0001 & as & $5(590.51)<10(688.35)=* * * 15(690.67)$ \\
\hline $\begin{array}{c}\text { Módulo de rotura - MOR } \\
(\mathrm{MPa})\end{array}$ & 0.0001 & as & $5(6.14)<10(9.38)=15(10.65)$ \\
\hline $\begin{array}{c}\text { Resistencia a la compresión } \\
\text { (MPa) }\end{array}$ & 0.0006 & as & $5(3.36)<10(6.20)=15(6.39)$ \\
\hline Extracción de tornillo - face (N) & 0.0085 & as & $5(159.40)<15(217.21)=10(226.97)$ \\
\hline $\begin{array}{c}\text { Extracción de tornillo - canto } \\
(\mathbf{N})\end{array}$ & 0.0003 & as & $5(96.16)<15(165.01)=10(210.66)$ \\
\hline Dureza Janka (N) & 0.0004 & as & $5(2441)<10(3541)=15(3552)$ \\
\hline Tracción perpendicular (MPa) & 0.0561 & $\mathrm{~s}$ & $5(0.27)<15(0.33)<10(0.40)$ \\
\hline
\end{tabular}

Legenda:

as - altamente significativo

$\mathrm{s}$ - significativo

* Dosis (valor medio de la propiedad medida)

** Nivel de confianza igual o superior al $94 \%$

*** El signo igual a $(=)$ que separa dos dosis significa que son estadísticamente iguales al nivel de significación $=95 \%$

(95\% de nivel de confianza)

Tabla 1 - Análisis de varianza simple de las propiedades medidas en los tableros prensados, en dependencia de la dosificación de pegamento $(5 \%, 10 \%$ y $15 \%)$ utilizada en su preparación 
Los resultados del ensayo no destructivo (END) posibilitaron la obtención de la velocidad del pulso de ultrasonido (VPU, en $\mathrm{m} / \mathrm{s}$ ) y permitieron detectar las diferencias aportadas a la estructura de las probetas por los diferentes contenidos de resina. Los datos de VPU, analizados e interpretados estadísticamente (test de Tukey al $5 \%$ de probabilidad) evidenciaron la superioridad de los contenidos de $10 \%(1897 \mathrm{~m} / \mathrm{s})$ y de $15 \%$ $(1992 \mathrm{~m} / \mathrm{s})$ con relación al contenido de 5\% (1561 $\mathrm{m} / \mathrm{s})$.

Debido a las particularidades del END (dimensiones de los tableros y frecuencia de resonancia de los emisores y receptores electroacústicos - de $45 \mathrm{kHz}$ ), no fue posible obtener informaciones más precisas de la heterogeneidad de los tableros

La constante elástica dinámica (CLL) obtenida en la dirección del largo fueron $1.48 \mathrm{GPa}, 2.34 \mathrm{GPa}$ e $2.59 \mathrm{GPa}$ para las dosificaciones de 5\%, 10\% y $15 \%$, respectivamente. Empleando el mismo método Gonçalves y Silva (2002) obtuvieron para tableros de partículas de Pinus valores de CLLen el rango de $1.69 \mathrm{GPa}$ al $4.59 \mathrm{GPa}$

No hubo correlación entre los resultados de la VPU y de la resistencia a la compresión longitudinal, contrariamente a los datos de la literatura para tableros manufacturados con la misma resina y partículas de madera (GONÇALVES; SILVA, 2002). Aparentemente, la compactación producida durante la manufactura de los tableros no garantiza la formación de rutas más favorables al desplazamiento de la onda de ultrasonido. No obstante, otras condiciones experimentales deberían ser evaluadas específicamente en la investigación con este tipo de tableros.

Considerándose que en la manufactura del tablero el costo de la resina es lo más elevado, y que no hubo diferencia estadística entre los contenidos de resina de $10 \%$ y de $15 \%$, fueron comparados los tableros con $10 \%$ de poliuretano con tableros de aglomerados comerciales. En la mayoría de las propiedades evaluadas los tableros comerciales presentaron los valores más elevados. Además, en el laboratorio no se logró alcanzar la misma metodología de fabricación de los tableros comerciales (temperatura y presión), e incluso se observó en los tableros comerciales la presencia de capas heterogéneas, lo que permitió un efecto más significativo en cohesión de las partículas.

La Tabla 2 muestra una comparación entre varios indicadores de calidad de aglomerados de la norma NBR 14810 (ABNT, 2002a, 2002b, 2002c) y aglomerados comerciales así como los obtenidos en el presente trabajo señalando entre paréntesis la dosis de poliuretano utilizada.

Las mayores diferencias entre ambos aglomerados están centradas en los índices de MOR, de la resistencia a la compresión longitudinal y de la extracción de tornillo en la cara y en el canto del tablero. Estas marcadas diferencias son atribuidas a la defectuosa manipulación del material a prensar sobre todo a la deficiente homogeneización de la distribución del poliuretano a través de la masa de material a prensar.

\begin{tabular}{c|c|c|c}
\hline Indicador & $\begin{array}{c}\text { Aglomerado con } \\
\text { poliuretano }\end{array}$ & $\begin{array}{c}\text { Aglomerado } \\
\text { comercial }\end{array}$ & $\begin{array}{c}\text { NBR 14810 } \\
\mathbf{( 2 0 0 2 )}\end{array}$ \\
\hline Densidad $\left(\mathbf{k g} / \mathbf{m}^{\mathbf{3}}\right)$ & $690(15=10)^{*}$ & 660 & \\
\hline Espesor $(\mathbf{m m})$ & $15.34(10)^{*}$ & 15.29 & $14 \mathrm{al} 20$ \\
\hline Hinchamiento en espesor & $5.03(15)<7.76(10)$ & 4.65 & 8.00 \\
\hline Dureza Janka (N) & $3587(10)^{*}$ & 3862 & \\
\hline MOR (MPa) & $10.65(15=10)^{*}$ & 22.85 & 16.00 \\
\hline Existencia a la compresión $(\mathbf{M P a})$ & $6.39(15=10)^{*}$ & 13.32 & \\
\hline Extracción de tornillo - face $\mathbf{( N )}$ & $227(10)$ & & 800 \\
\hline
\end{tabular}

* Dosis (\%) de poliuretano utilizada

Tabla 2 - Indicadores de calidad de aglomerados según la norma NBR 14810, comparados con los aglomerados comerciales y aquellos obtenidos en el presente trabajo usando poliuretano como aglomerante 


\section{Conclusiones}

De acuerdo con las condiciones en que esta investigación fue conducida, y después de efectuarse el análisis estadístico fue posible concluir que:

(a) el contenido de resina presentó un efecto significativo en todas las propiedades evaluadas, con excepción de los valores de tracción perpendicular;

(b) no hubo diferencia significativa en las propiedades de los tableros para los contenidos de resina del $10 \%$ y del $15 \%$, ambos superiores estadísticamente al contenido del 5\%;

(c) los tableros de aglomerados comerciales fueron superiores a los tableros con $10 \%$ de resina, con excepción de la dureza Janka (no hubo diferencia) e hinchamiento después de 24 h (los tableros con $10 \%$ presentaron un valor menor);

(d) la norma utilizada presenta valores mínimos recomendados solamente para los ensayos de flexión estática, de extracción de tornillo, de hinchamiento, de absorción de agua y de tracción perpendicular. Los tableros con resina a base de aceite de ricino no alcanzaron los valores mínimos exigidos en la norma NBR 14810 (ABNT, 2002a, 2002 b, 2002c) y de este modo las aplicaciones para los tableros son restringidas a la mueblería y a los ambientes protegidos de las intemperies; $y$

(e) la distribución del tamaño de las partículas de bambú y de la razón largo/espesor, las cantidades de poliól y del pre-polímero empleados en la síntesis de la resina, la densidad del tablero, el contenido de resina, el espesor del tablero, o la utilización de aditivos como la parafina, son las alternativas que deben ser investigadas para mejorar el desempeño de los tableros.

\section{Referencias}

\section{ASSOCIAÇÃO BRASILEIRA DE NORMAS} TÉCNICAS. NBR 14810: chapas de madeira aglomerada: parte 1: terminologia. Rio de Janeiro, 2002. 5 p.

\section{ASSOCIAÇÃO BRASILEIRA DE NORMAS} TÉCNICAS. NBR 14810: chapas de madeira aglomerada: parte 2: requisitos. Rio de Janeiro, 2002. 4 p.

\section{ASSOCIAÇÃO BRASILEIRA DE NORMAS} TÉCNICAS. NBR 14810: chapas de madeira aglomerada: parte 3: métodos de ensaio. Rio de Janeiro, 2002. 27 p.
ASSOCIAÇÃO BRASILEIRA DA INDÚSTRIA DE PAINÉIS DE MADEIRA. ABIPA. Disponível em: < www.abipa.com.br>. Acesso em: 01 ago. 2010.

AZAMBUjA, M. A. Avaliação do Adesivo Poliuretano a Base de Mamona para Fabricação de Madeira Laminada Ccolada (MLC). 115 f. 2002. Dissertação (Mestrado em Engenharia Civil) - Escola de Engenharia, Universidade de São Paulo, São Carlos, 2002.

CAMPOS, C. I.; LAHR, F. A. R. Caracterización del MDF Producido a Partir de Eucalipto y Adhesivo Poliuretano Natural. Madera: Ciencia y Tecnología, Concepción, Chile, v. 6, n. 1, p. 7384, 2004.

DIAS, F. M.; LAHR, F. A. R. Alternative Castor Oil-Based Polyurethane Adhesive Used in the Production of Plywood. Materials Research, São Carlos, v. 7, n.3, p. 413-420, 2004.

DIAS, F. M et al. Painéis de Partículas de Eucalyptus Manufaturadas com Resina Poliuretana a Base de Mamona. In: CONGRESSO BRASILEIRO EM MADEIRAS E EM ESTRUTURAS DE MADEIRA, 9., Cuiabá, 2004. Anais... Cuiabá, 2004. 1 CD-ROM.

\section{ELEOTÉRIO, J. R. Propriedades Físicas e}

Mecânicas de Painéis MDF de Diferentes Densidades e Teores de Resina. 2000. 120 f. Dissertação (Mestrado em Ciência e Tecnologia de Madeiras) - Escola Superior de Agricultura, Universidade de São Paulo, Piracicaba, 2000.

FIORELLI, J. Utilização de Fibras de Carbono e de Fibras de Vidro para Reforço de Vigas de Madeira. 164 f. 2002. Dissertação (Mestrado em Engenharia) - Escola de Engenharia, Universidade de São Paulo, São Carlos, 2002.

GONÇALVES, R.; SILVA, S. A. M. 2002. Avaliação de Chapas de Fibra de Madeira Utilizando Ultra-Som. In: CONGRESSO IBERO-AMERICANO DE PESQUISA E DESENVOLVIMENTO DE PRODUTOS FLORESTAIS, 2.; SEMINÁRIO EM TECNOLOGIA DA MADEIRA E PRODUTOS FLORESTAIS NÃO-MADEIRÁVEIS, 1., 2002, Curitiba. Anais... Curitiba, PR, 2002. 1 CD-ROM.

ITAPAGÉ. Indústria de Celulose e Papel. 2004. Disponível em: <www.itapage.com>. Acesso em: 25 abr. 2004. 
JESUS, M. H. Estudo do Adesivo Poliuretano à Base de Mamona em Madeira Laminada Colada (MLC). 109 f. 2000. Tese (Doutorado em Engenharia Civil) - Escola de Engenharia, Universidade de São Paulo, São Carlos, 2000.

KEHL Indústria e Comércio. 2004. Disponível em: <www.kehl.ind.br>. Acesso em: 21 abr. 2004.

KOLLMANN, F. P.; KUENZI, E. W.; STAMM, S. Principles of Wood Science and Technology: wood-based materials, properties of particleboard. New York: Nail-Holding and Screw-Holding Ability, 1975. p. 523-529.

MALONEY, T. M. 1996. The Family of Wood Composite Materials. Forest Products Journal, v. 46, n. 2, p.19-26, 1996

MOTHÉ C. G.; ARAÚJO C. R. Caracterização Térmica e Mecânica de Compósitos de Poliuretano com Fibras de Curauá. Polímeros: Ciência e Tecnologia, v. 14, n. 4, p. 274-278, 2004.

NASCIMENTO, M. F. Chapas de Partículas Homogêneas: madeiras do nordeste do Brasil. 125 f. 2003. Tese (Doutorado em Engenharia Civil) Escola de Engenharia, Universidade de São Paulo, São Carlos, 2003.
PEREIRA, M. A. R.; BERALDO, A. L. Bambu de Corpo e Alma. Bauru, SP: Canal 6 Editora, 2007. $240 \mathrm{p}$.

PIZZI, A. Advanced Wood Adhesives Technology. New York: Marcel Dekker, 1994. 297 p.

SOCIEDADE BRASILEIRA DE

SILVICULTURA. 2004. Disponível em:

<www.sbs.org.br>. Acesso em: 22 maio 2005.

\section{Agradecimientos}

Los autores agradecen al $\mathrm{CNPq}$ por las becas, a la empresa Kehl Polímeros por la donación del adhesivo, al LAMEM de San Carlos y en especial a la investigadora Fátima Nascimento, por la fabricación de los tableros y a los laboratorios del SENAI, Itatiba, y del Centro de Tecnología, Unicamp, por la ejecución de los ensayos mecánicos de los tableros. Agradecen también al Prof. Luis Ernesto Brossard por el análisis estadístico de los datos. 